\title{
Uniqueness for nonlinear degenerate problems
}

\author{
Noureddine IGBIDA* \\ C.M.A.F./Universidade de Lisboa \\ Av. Prof. Gama Pinto, 2 \\ 1649-003 Lisboa, Portugal \\ e-mail: igbida@lmc.fc.ul.pt. \\ José Miguel URBANO** \\ Departamento de Matemática \\ Universidade de Coimbra \\ 3001-454 Coimbra, Portugal \\ e-mail: jmurb@mat.uc.pt.
}

\begin{abstract}
We prove existence and uniqueness results for weak solutions of nonlinear degenerate problems arising in various physical models. The main novelty in the article concerns the uniqueness, which employs a technique based in showing that weak solutions are also entropy solutions, for which uniqueness follows from a straightforward adaptation of known results. We treat equations with lower order terms that have a particular structure and show with a counterexample that for general lower order terms the uniqueness does not hold.
\end{abstract}

2000 Mathematics Subject Classification: 35D99, 35K65, 35R35.

Key words: Degenerate parabolic equations, uniqueness, weak solutions, entropy inequalities.

\section{Introduction}

This article deals with the question of establishing the uniqueness of weak solutions for boundary value problems involving doubly nonlinear degenerate equations. Besides having a mathematical interest in their own right, equations of this type also arise as models for a variety of diffusion phenomena. Consider, for example,

${ }^{*}$ Research supported by CMAF/FCT.

** Research supported by CMUC/FCT and Praxis XXI. 
the problem

$$
\begin{cases}u_{t}-\nabla \cdot G(u)-h \in \Delta_{p} \varphi(u) & \text { in } Q:=(0, T) \times \Omega \\ \varphi(u)=0 & \text { on } \Sigma:(0, T) \times \Gamma \\ u(0, .)=u_{0}(.) & \text { in } \Omega,\end{cases}
$$

where $\Omega$ is a bounded domain with a regular boundary $\Gamma, T>0$, the operator $\Delta_{p} w=\nabla \cdot\left(|\nabla w|^{p-2} \nabla w\right)$ is the $p$-Laplacian, $\varphi$ is a maximal monotone graph in $\mathbb{R}$ and $G$ is a vector function. A large field of applications corresponds to the case of a continuous $\varphi$, for which (1.1) models free boundary problems involving a solid-liquid phase change of Stefan type. Another interesting application arises when the inverse $\gamma:=\varphi^{-1}$ is continuous. Then (1.1) is a mathematical model for saturated-unsaturated flow of water through a porous medium and falls into the scope of general initial boundary value problems of the form

$$
\begin{cases}\gamma(v)_{t}=\nabla \cdot \mathbf{a}(\nabla v, \gamma(v))+h & \text { in } Q:=(0, T) \times \Omega \\ v=0 & \text { on } \Sigma:(0, T) \times \Gamma \\ \gamma(v(0, .))=u_{0}(.) & \text { in } \Omega\end{cases}
$$

where $\mathbf{a}:(\xi, z) \in \mathbb{R}^{N} \times \mathbb{R} \rightarrow \mathbb{R}^{N}$ is continuous and monotone in $\xi$. Problems of this type are called elliptic-parabolic and have been extensively studied in the literature under general assumptions on a (cf. $[1,3,12,9]$ and the references therein). In fact, assuming that $\gamma$ is non decreasing, it is well known that, for any $u_{0} \in L^{\infty}(\Omega)$ and $h \in L^{\infty}(Q),(1.2)$ is well posed in the sense of Hadamard when solutions are taken in the weak sense. On the contrary, the problem of establishing the existence and uniqueness of weak solutions of (1.1) seems to be complicated and is poorly investigated in general. The equation in (1.1) has a hyperbolic character in the set where $\varphi(u)=0$, and we say that (1.1) is of parabolic-hyperbolic type; in general, uniqueness of a weak solution does not hold (see Remark 4). In his recent paper [6], Carrillo proves that problems of type (1.1) are well posed using the concept of "entropy solutions", which are weak solutions that satisfy some additional conditions called entropy conditions. We will be interested in the (existence and) uniqueness of a weak solution of (1.1) in the case of a continuous and non decreasing $\varphi$ and under the additional structure condition

$$
G(r)=F(\varphi(r))
$$

in other words, we will assume that the convective term is independent of the jumps of $\varphi^{-1}$. In this case, (1.1) is a doubly nonlinear degenerate parabolic problem for which the existence of a weak solution follows from the general results obtained in [1], using variational techniques, and [10], using Euler-implicit approximation; here we revisit the existence through nonlinear semigroup theory. But our main concern is the uniqueness, that was still an open question. We present a new approach inspired in the article [6] of Carrillo and consisting in showing that every weak solution satisfies appropriate entropy inequalities; then the 
$L^{1}-$ contraction for weak solutions is established in exactly the same way as for the case $p=2$. The main application we have in mind concerns the Stefan problem for the $p$-Laplacian. Observe that the equation in (1.1), with the assumption (1.3), can also be written in the form

$$
\partial_{t} \gamma(\theta)-\Delta_{p} \theta-\nabla \cdot F(\theta) \ni h,
$$

with $\gamma=\varphi^{-1}$ (possibly) a maximal monotone graph, which clearly includes the Stefan problem for the $p$-Laplacian with a temperature dependent convective term, for example, for $F(\theta)=\theta \mathbf{v}$, and $\mathbf{v}$ a given constant vector. This problem was treated in [14] and [15], for $\mathbf{v}$ not necessarily constant, from the point of view of, respectively, the existence of a weak solution (in the more general case of a convective term depending on the enthalpy) and its continuity. We now complete the study establishing the uniqueness. We recall that the problem is completely solved in the case $p=2$. In fact, the linearity of the Laplacian, enables one to interpret the difference of the equations for two solutions $u_{1}$ and $u_{2}$ as a linear parabolic equation in $u_{1}-u_{2}$ and then to solve the dual equation, thus proving that

$$
\int_{\Omega}\left|u_{1}(t)-u_{2}(t)\right| \leq \int_{\Omega}\left|u_{01}-u_{02}\right|+\int_{0}^{t} \int_{\Omega}\left|h_{1}-h_{2}\right|
$$

(see [11] and [13]). Another approach, valid only for the case $F \equiv 0$, is due to Brézis and Crandall [5] and their idea is to apply the operator $(I d-\varepsilon \Delta)^{-1}$ to the difference of the equations. We stress that both approaches strongly use the linearity of the Laplacian and are not applicable to (1.1), for $1<p<2$ or $2<p<\infty$. As mentioned above, we will also be interested in revisiting the existence of a solution to (1.1) through nonlinear semigroup theory in $L^{1}(\Omega)$. A crucial question here is the well posedness and the contraction properties in $L^{1}(\Omega)$ of the stationary problem

$$
\begin{cases}u-\Delta_{p} \varphi(u)-\nabla \cdot F(\varphi(u))=f & \text { in } \Omega \\ \varphi(u)=0 & \text { on } \Gamma\end{cases}
$$

with $f \in L^{\infty}$. Assuming that $\varphi$ is continuous and non decreasing and (1.3) for a Lipschitz $F$, we start, in section 2 , by the study of (1.4). We first establish the uniqueness for $L^{1}$ data and then the existence for $L^{\infty}$ data. This paves the way to section 3, where we obtain the uniqueness and the existence of a weak solution of (1.1), belonging additionally to $C\left([0, T) ; L^{1}(\Omega)\right)$. In fact, we will use the same ideas of section 2 to establish the uniqueness for $L^{1}$ data and then we prove that solutions in the sense of semigroups (mild solutions) are weak solutions of (1.1) if the data are in $L^{\infty}$. We close with a counterexample for the uniqueness in the case when (1.3) is relaxed. 


\section{The stationary problem}

In order to study the problem in the framework of nonlinear semigroup theory, we consider, in this section, the stationary problem associated with (1.1)-(1.3). To simplify the notation, let's introduce the vector function

$$
\mathbf{a}(\xi, s)=|\xi|^{p-2} \xi+F(s), \quad \xi \in \mathbb{R}^{N}, s \in \mathbb{R}
$$

so that the stationary problem reads

$$
\left\{\begin{array}{ll}
u-\nabla \cdot \mathbf{a}(\nabla \varphi(u), \varphi(u))=f & \text { in } \Omega \\
\varphi(u)=0 & \text { on } \Gamma
\end{array} .\right.
$$

Introduce the function

$$
\varphi_{0}^{-1}(x)=\min \left\{\varphi^{-1}(x)\right\}
$$

and define the set

$$
E=\left\{r \in \mathbb{R}: \varphi_{0}^{-1} \text { is discontinuous at } r\right\} .
$$

We state the assumptions on the data that will hold throughout the paper:

$$
\begin{aligned}
& \varphi \in \mathcal{C}(\mathbb{R}) \text { is non decreasing, with } \varphi(0)=0, \\
& \text { and } 0 \text { is not an accumulation point of } E \text {. } \\
& F: \mathbb{R} \longrightarrow \mathbb{R}^{N} \text { is Lipschitz, with } F(0)=\mathbf{0} .
\end{aligned}
$$

The main theorem of this section is

Theorem 1 Let $f \in L^{\infty}(\Omega)$. Then there exists a unique weak solution u of $\mathrm{S}(\mathrm{f})$ in the sense that

$$
\left\{\begin{array}{l}
u \in L^{\infty}(\Omega), \varphi(u) \in W_{0}^{1, p}(\Omega) \\
u-\nabla \cdot \mathbf{a}(\nabla \varphi(u), \varphi(u))=f \quad \text { in } \quad \mathcal{D}^{\prime}(\Omega)
\end{array} .\right.
$$

Moreover

$$
\|u\|_{\infty} \leq\|f\|_{\infty}
$$

and, for any $f_{i} \in L^{\infty}(\Omega)$ and $u_{i}$ weak solutions of $S\left(f_{i}\right), i=1,2$,

$$
\left\|\left(u_{1}-u_{2}\right)^{+}\right\|_{1} \leq\left\|\left(f_{1}-f_{2}\right)^{+}\right\|_{1} .
$$




\subsection{Proof of the uniqueness}

We start the proof by the uniqueness, establishing it in a slightly more general setting. We introduce the following notation that will be used throughout the paper:

$$
\begin{gathered}
\operatorname{Sign}^{+}(s)= \begin{cases}1 & \text { if } s>0 \\
{[0,1]} & \text { if } s=0, \\
0 & \text { if } s<0\end{cases} \\
\operatorname{Sign}_{0}^{+}(s)=\left\{\begin{array}{lll}
1 & \text { if } s>0 \\
0 & \text { if } & s \leq 0
\end{array} \quad \operatorname{Sign}_{\text {max }}^{+}(s)=\left\{\begin{array}{lll}
1 & \text { if } s \geq 0 \\
0 & \text { if } & s<0
\end{array}\right.\right.
\end{gathered} \text { and } .
$$

The uniqueness for the stationary problem is an immediate corollary of

Proposition 1 Let $f_{1}, f_{2} \in L^{1}(\Omega)$. If $u_{i}$ is a weak solution of $S\left(f_{i}\right)$ for $i=1,2$, then

$$
\left\|\left(u_{1}-u_{2}\right)^{+}\right\|_{1} \leq\left\|\left(f_{1}-f_{2}\right)^{+}\right\|_{1} .
$$

The proof will follow as a corollary of a sequence of lemmas that we next present. The basic idea in the lemmas is to show that a weak solution of $\mathrm{S}(\mathrm{f})$ satisfies entropy inequalities of the type

$$
\int_{\Omega} \operatorname{Sign}_{0}^{+}(u-k)\{(u-f) \xi+\mathbf{a}(\nabla \varphi(u), \varphi(u)) \cdot \nabla \xi\} \leq 0
$$

for appropriate choices of $k$ and $\xi$. Assume that $\varphi^{-1}(0)=[m, M]$, with $m<M$. The reason to choose $k$ and $\xi$ is determined by the fact that the function

$$
\phi=H_{\epsilon}(\varphi(u)-\varphi(k)) \xi
$$

where $H_{\epsilon}$ is the approximation of $\operatorname{Sign}^{+}$defined by

$$
H_{\epsilon}(r)=\min \left(\frac{r^{+}}{\varepsilon}, 1\right)
$$

is to be chosen as test function in the problem. It is clear that if $\xi$ vanishes on the boundary, the same happens with $\phi$ but if that is not the case then we must have $H_{\epsilon}(-\varphi(k))=0$, i.e. $\varphi(k) \geq 0$, which follows if $k \geq m$. We first derive the entropy inequalities for constants $k$ such that $\varphi(k) \notin E$. Then we obtain them for the extreme points of the intervals where $\varphi$ is constant and apply a "filling" lemma which says that if the entropy inequalities are valid for $k_{1}$ and $k_{2}$, with $\varphi\left(k_{1}\right)=\varphi\left(k_{2}\right) \in E$, then they are also valid for any $k \in\left[k_{1}, k_{2}\right]$. This is enough to cover the case $k \in \mathbb{R}, \xi \in W_{0}^{1, p}(\Omega)$. But when $\xi \in W^{1, p}(\Omega)$ then we must choose $k \geq m$ and we have problems with the $k$ 's such that $\varphi(k)=0$ since we can not use the filling lemma without the entropy inequality for $k=m$. The idea is then 
to deal with functions $z$ (instead of constants $k$ ) vanishing on the boundary of $\Omega$, choosing a sequence $z_{n}$ such that $z_{n}<m$ and $z_{n} \rightarrow m$, thus approximating the infimum $m$ of the interval where $\varphi \equiv 0$. The idea here is that $z_{n}$ may be negative since now it is the fact that they vanish on the boundary of $\Omega$ that guarantees that (2.4) is a good test function.

Lemma 1 Let $f \in L^{1}(\Omega)$ and $u$ be a weak solution of $\mathrm{S}(\mathrm{f})$. Then

$$
\begin{aligned}
& \int_{\Omega} \operatorname{Sign}_{0}^{+}(u-z)\{(u-f) \xi+[\mathbf{a}(\nabla \varphi(u), \varphi(u))-\mathbf{a}(\nabla \varphi(z), \varphi(z))] \cdot \nabla \xi\} \\
& \quad \leq \int_{\Omega} \operatorname{Sign}_{0}^{+}(u-z) \xi \nabla \cdot \mathbf{a}(\nabla \varphi(z), \varphi(z)),
\end{aligned}
$$

for all pairs $(z, \xi) \in L^{\infty}(\Omega) \times\left[W^{1, p}(\Omega) \cap L^{\infty}(\Omega)\right]$ such that $\xi \geq 0, \varphi(z) \in W^{1, p}(\Omega)$, $\varphi(z) \notin E$ a.e. in $\Omega, \nabla \cdot \mathbf{a}(\nabla \varphi(z), \varphi(z)) \in L^{1}(\Omega)$ and $(\varphi(u)-\varphi(z))^{+} \xi=0$ on $\Gamma$.

Proof. Let $z$ and $\xi$ be as in the statement of the lemma; then

$$
H_{\epsilon}(\varphi(u)-\varphi(z)) \xi \in W_{0}^{1, p}(\Omega) \cap L^{\infty}(\Omega)
$$

so it is an admissible test function in (2.3) and

$$
\begin{aligned}
& \int_{\Omega} H_{\epsilon}(\varphi(u)-\varphi(z))(u-f) \xi \\
& \quad+\int_{\Omega} \mathbf{a}(\nabla \varphi(u), \varphi(u)) \cdot \nabla\left[H_{\epsilon}(\varphi(u)-\varphi(z)) \xi\right]=0 .
\end{aligned}
$$

It is obvious that, as $\epsilon \rightarrow 0$, the first term of (2.5) converges to

$$
\int_{\Omega} \operatorname{Sign}_{0}^{+}(\varphi(u)-\varphi(z))(u-f) \xi
$$

As to the second term, note that

$$
\begin{aligned}
\int_{\Omega} & \mathbf{a}(\nabla \varphi(u), \varphi(u)) \cdot \nabla\left[H_{\varepsilon}(\varphi(u)-\varphi(z)) \xi\right] \\
= & \int_{\Omega}[\mathbf{a}(\nabla \varphi(u), \varphi(u))-\mathbf{a}(\nabla \varphi(z), \varphi(z))] \cdot \nabla\left[H_{\varepsilon}(\varphi(u)-\varphi(z)) \xi\right] \\
& \quad+\int_{\Omega} \mathbf{a}(\nabla \varphi(z), \varphi(z)) \cdot \nabla\left[H_{\varepsilon}(\varphi(u)-\varphi(z)) \xi\right] \\
= & I_{\epsilon}^{1}+I_{\epsilon}^{2} .
\end{aligned}
$$

Clearly

$$
I_{\epsilon}^{2} \longrightarrow-\int_{\Omega} \operatorname{Sign}_{0}^{+}(\varphi(u)-\varphi(z)) \xi \nabla \cdot \mathbf{a}(\nabla \varphi(z), \varphi(z))
$$


and, concerning $I_{\epsilon}^{1}$, using a well known inequality,

$$
\begin{aligned}
I_{\epsilon}^{1} \geq & \int_{\Omega} H_{\varepsilon}(\varphi(u)-\varphi(z))[\mathbf{a}(\nabla \varphi(u), \varphi(u))-\mathbf{a}(\nabla \varphi(z), \varphi(z))] \cdot \nabla \xi \\
& +\int_{\Omega} H_{\varepsilon}^{\prime}(\varphi(u)-\varphi(z)) \xi(F(\varphi(u))-F(\varphi(z))) \cdot \nabla(\varphi(u)-\varphi(z)) .
\end{aligned}
$$

Consider now the last term in (2.6) and call it $I_{\epsilon}$. Using (2.2), we have, denoting by $C$ the Lipschitz constant of $F$,

$$
\begin{aligned}
\left|I_{\epsilon}\right| & \leq \int_{\Omega}|F(\varphi(u))-F(\varphi(z))| H_{\varepsilon}^{\prime}(\varphi(u)-\varphi(z))|\nabla(\varphi(u)-\varphi(z))| \xi \\
& \leq C \int_{[0 \leq|\varphi(u)-\varphi(z)| \leq \epsilon]}|\varphi(u)-\varphi(z)| \frac{1}{\epsilon}|\nabla(\varphi(u)-\varphi(z))| \xi \\
& \leq C \int_{[0 \leq|\varphi(u)-\varphi(z)| \leq \epsilon]}|\nabla(\varphi(u)-\varphi(z))| \xi \longrightarrow 0 \text { as } \epsilon \rightarrow 0
\end{aligned}
$$

so that

$$
\liminf _{\epsilon \rightarrow 0} I_{\epsilon}^{1} \geq \int_{\Omega} \operatorname{Sign}_{0}^{+}(\varphi(u)-\varphi(z))[\mathbf{a}(\nabla \varphi(u), \varphi(u))-\mathbf{a}(\nabla \varphi(z), \varphi(z))] \cdot \nabla \xi .
$$

At last, letting $\varepsilon \rightarrow 0$ in $(2.5)$, one gets

$$
\begin{aligned}
\int_{\Omega} & \operatorname{Sign}_{0}^{+}(\varphi(u)-\varphi(z))\{(u-f) \xi \\
& +[\mathbf{a}(\nabla \varphi(u), \varphi(u))-\mathbf{a}(\nabla \varphi(z), \varphi(z))] \cdot \nabla \xi\} \\
\leq & \int_{\Omega} \operatorname{Sign}_{0}^{+}(\varphi(u)-\varphi(z)) \xi \nabla \cdot \mathbf{a}(\nabla \varphi(z), \varphi(z))
\end{aligned}
$$

and, since

$$
\operatorname{Sign}_{0}^{+}(\varphi(u)-\varphi(z))=\operatorname{Sign}_{0}^{+}(u-z)
$$

because $\varphi(z) \notin E$, the result follows.

The next lemma concerns an approximation that will be useful in the sequel.

Lemma 2 If $m=\inf \{r \in \mathbb{R}: \varphi(r)=0\}>-\infty$, then there exists $z_{n} \in L^{\infty}(\Omega)$, with $\varphi\left(z_{n}\right) \in W_{0}^{1, p}(\Omega)$, such that $z_{n}<m$ a.e. in $\Omega, \varphi\left(z_{n}\right) \notin E$ and, as $n \rightarrow \infty$, $z_{n} \rightarrow m$ in $L^{1}(\Omega), \nabla \cdot \mathbf{a}\left(\nabla \varphi\left(z_{n}\right), \varphi\left(z_{n}\right)\right) \rightarrow 0$ in $L^{1}(\Omega)$ and $\mathbf{a}\left(\nabla \varphi\left(z_{n}\right), \varphi\left(z_{n}\right)\right) \rightarrow 0$ in $\left[L^{p^{\prime}}(\Omega)\right]^{N}$-weak.

Proof. We use the strong maximum principal of Vazquez (cf. Theorem 5 of [16]) in the following way. Take a sequence of real numbers $c_{n} \leq 0$ such that $c_{n} \nearrow 0$ and consider $w_{n} \in W_{0}^{1, p}(\Omega) \cap L^{\infty}(\Omega)$ such that

$$
\beta\left(w_{n}\right)-\Delta_{p} w_{n}=-c_{n} \quad \text { in } \quad \mathcal{D}^{\prime}(\Omega),
$$


where $\beta(r)=r^{p}, r \in[0, \infty)$. It is clear that $\beta$ satisfies the assumptions of Theorem 5 in [16]. Since $w_{n} \in C^{1}(\Omega), \Delta_{p} w_{n} \in L^{\infty}(\Omega), w_{n} \geq 0$ and $\Delta_{p} w_{n} \leq \beta\left(w_{n}\right)$, a.e. in $\Omega$, the conclusion of the theorem is that $w_{n}>0$ in $\Omega$. Then define $z_{n}=$ $\varphi^{-1}\left(-w_{n}\right) \in L^{\infty}(\Omega)$. It is obvious that $z_{n}<m$, a.e. in $\Omega$ and $\varphi\left(z_{n}\right) \in W_{0}^{1, p}(\Omega)$. Moreover, by well known results,

$$
\left\|\beta\left(w_{n}\right)\right\|_{\infty} \leq\left|c_{n}\right| \quad \text { which implies } \quad\left\|w_{n}\right\|_{\infty} \leq\left|c_{n}\right|^{\frac{1}{p}}
$$

and so $w_{n} \rightarrow 0$ uniformly. Since 0 is not an accumulation point of $E$ by assumption (2.1), we have that $\varphi\left(z_{n}\right)=-w_{n} \notin E$. Finally, observing that, as $c_{n} \nearrow 0, w_{n} \rightarrow 0$, we easily get $z_{n} \rightarrow m$ in $L^{1}(\Omega), \nabla w_{n} \rightarrow 0$ in $L^{1}(\Omega)$ and

$$
\begin{aligned}
\nabla \cdot \mathbf{a}\left(\nabla \varphi\left(z_{n}\right), \varphi\left(z_{n}\right)\right) & =-\Delta_{p} w_{n}+\nabla \cdot F\left(-w_{n}\right) \\
& =-\beta\left(w_{n}\right)-c_{n}-F^{\prime}\left(-w_{n}\right) \cdot \nabla w_{n} \rightarrow 0 \quad \text { in } L^{1}(\Omega) .
\end{aligned}
$$

To obtain the missing assertion we employ the usual monotonicity techniques.

The last lemma of this section establishes the entropy inequalities for values of $k$ in the interior of the plane section at the zero level.

Lemma 3 Assume that $0 \in E$ and $\varphi^{-1}(0)=[m, M], m<M$. Let $f \in L^{1}(\Omega)$ and $u$ be a weak solution of $S(f)$. Then

$$
\int_{\Omega} \operatorname{Sign}_{0}^{+}(u-k)\{(u-f) \xi+\mathbf{a}(\nabla \varphi(u), \varphi(u)) \cdot \nabla \xi\} \leq 0,
$$

for all pairs $(k, \xi) \in \mathbb{R} \times\left[W^{1, p}(\Omega) \cap L^{\infty}(\Omega)\right]$ such that $m \leq k \leq M$ and $\xi \geq 0$.

Proof. To start with, as in the proof of Lemma 1, one sees that taking $H_{\varepsilon}(\varphi(u)) \xi$ as a test function in (2.3), with $\xi \in W^{1, p}(\Omega)$ such that $\xi \geq 0$ and letting $\varepsilon \rightarrow 0$, one gets

$$
\int_{\Omega} \operatorname{Sign}_{0}^{+}(\varphi(u))\{(u-f) \xi+\mathbf{a}(\nabla \varphi(u), \varphi(u)) \cdot \nabla \xi\} \leq 0 .
$$

Since $\operatorname{Sign}_{0}^{+}(\varphi(u))=\operatorname{Sign}_{0}^{+}(u-M), u$ satisfies

$$
\int_{\Omega} \operatorname{Sign}_{0}^{+}(u-M)\{(u-f) \xi+\mathbf{a}(\nabla \varphi(u), \varphi(u)) \cdot \nabla \xi\} \leq 0 .
$$

On the other hand, consider $z_{n}$ as given in Lemma 2 and use Lemma 1 to get

$$
\begin{aligned}
& \int_{\Omega} \operatorname{Sign}_{0}^{+}\left(u-z_{n}\right)\left\{(u-f) \xi+\left[\mathbf{a}(\nabla \varphi(u), \varphi(u))-\mathbf{a}\left(\nabla \varphi\left(z_{n}\right), \varphi\left(z_{n}\right)\right)\right] \cdot \nabla \xi\right\} \\
& \quad \leq \int_{\Omega} \operatorname{Sign}_{0}^{+}\left(u-z_{n}\right) \xi \nabla \cdot \mathbf{a}\left(\nabla \varphi\left(z_{n}\right), \varphi\left(z_{n}\right)\right) .
\end{aligned}
$$


It is obvious that

$$
\operatorname{Sign}_{0}^{+}\left(u-z_{n}\right) \longrightarrow \operatorname{Sign}_{\max }^{+}(u-m)
$$

and in addition

$$
\operatorname{Sign}_{m a x}^{+}(u-m) \mathbf{a}(\nabla \varphi(u), \varphi(u)) \cdot \nabla \xi=\operatorname{Sign}_{0}^{+}(u-M) \mathbf{a}(\nabla \varphi(u), \varphi(u)) \cdot \nabla \xi,
$$

so letting $n \rightarrow \infty$ in (2.8), one obtains

$$
\int_{\Omega} \operatorname{Sign}_{m a x}^{+}(u-m)(u-f) \xi+\operatorname{Sign}_{0}^{+}(u-M) \mathbf{a}(\nabla \varphi(u), \varphi(u)) \cdot \nabla \xi \leq 0 .
$$

Now, as in the proof of Theorem 3 of [6], we apply Lemma 2 (in fact, its non Hilbertian version, for which the proof is similar and left to the reader) of [6] with

$$
\left\{\begin{array}{l}
\mathcal{O}=\Omega, e=0, F=\operatorname{Sign}_{0}^{+}(u-M) \mathbf{a}(\nabla \varphi(u), \varphi(u)) \\
G_{1}=\operatorname{Sign}_{0}^{+}(u-M)(u-f) \text { and } G_{2}=\operatorname{Sign}_{m a x}^{+}(u-m)(u-f)
\end{array}\right.
$$

obtaining

$$
\begin{aligned}
& \int_{\Omega}(u-f)\left(\operatorname{Sign}_{0}^{+}(u-M)(1-\tilde{H}(u-k))+\operatorname{Sign}_{m a x}^{+}(u-m) \tilde{H}(u-k)\right) \xi \\
& \quad+\operatorname{Sign}_{0}^{+}(u-M) \mathbf{a}(\nabla \varphi(u), \varphi(u)) \cdot \nabla \xi \leq 0,
\end{aligned}
$$

for all $k \in[m, M]$ and for some $\tilde{H}(u-k) \in \operatorname{Sign}^{+}(u-k)$. It follows, due to the facts that

$$
\begin{gathered}
\operatorname{Sign}_{0}^{+}(u-M)(1-\tilde{H}(u-k))=0, \\
\operatorname{Sign}_{m a x}^{+}(u-m) \tilde{H}(u-k)=\tilde{H}(u-k)
\end{gathered}
$$

and

$$
\operatorname{Sign}_{0}^{+}(u-M) \mathbf{a}(\nabla \varphi(u), \varphi(u))=\operatorname{Sign}_{0}^{+}(u-k) \mathbf{a}(\nabla \varphi(u), \varphi(u))
$$

that

$$
\int_{\Omega}(u-f) \tilde{H}(u-k) \xi+\operatorname{Sign}_{0}^{+}(u-k) \mathbf{a}(\nabla \varphi(u), \varphi(u)) \cdot \nabla \xi \leq 0
$$

To conclude take, for any $k \in[m, M)$ a sequence $\left(k_{n}\right)_{n \in \mathbb{N}}$ such that $m<k<k_{n}<$ $M$ and $k_{n} \downarrow k$. Then $\tilde{H}\left(u-k_{n}\right) \uparrow \operatorname{Sign}_{0}^{+}(u-k)$ and $\operatorname{Sign}_{0}^{+}\left(u-k_{n}\right) \uparrow \operatorname{Sign}_{0}^{+}(u-k)$ a.e. in $\Omega$; hence replacing $k$ by $k_{n}$ in (2.9) and letting $n \rightarrow \infty$, one gets

$$
\int_{\Omega} \operatorname{Sign}_{0}^{+}(u-k)\{(u-f) \xi+\mathbf{a}(\nabla \varphi(u), \varphi(u)) \cdot \nabla \xi\} \leq 0
$$

which ends up the proof. 
We can finally prove the main proposition that yields the uniqueness as an obvious corollary.

Proof of Proposition 1. Since weak solutions are bounded, we may assume that $\varphi^{-1}(r)$ is a bounded interval for all $r \in E$. Applying Lemmas 1 and 3 we get

$$
\int_{\Omega} \operatorname{Sign}_{0}^{+}(u-k)\{(u-f) \xi+\mathbf{a}(\nabla \varphi(u), \varphi(u)) \cdot \nabla \xi\} \leq 0,
$$

for any $(k, \xi) \in \mathbb{R} \times\left[W_{0}^{1, p}(\Omega) \cap L^{\infty}(\Omega)\right]$ such that $\xi \geq 0$ and $\varphi(k) \notin E$ or $\varphi(k)=0$; and for any $(k, \xi) \in \mathbb{R}^{+} \times\left[W^{1, p}(\Omega) \cap L^{\infty}(\Omega)\right]$ such that $\xi \geq 0$ and $\varphi(k) \notin E$ or $\varphi(k)=0$. For $k \in \mathbb{R}$ such that $\varphi(k) \in E$ and $\varphi(k) \neq 0$ we use the same arguments of [6] (cf. Theorem 3 there) to prove (2.10) for any $\xi \in W_{0}^{1, p}(\Omega)$ such that $\xi \geq 0$ and, if $k \geq 0, \varphi(k) \in E$ and $\varphi(k) \neq 0$, for any $\xi \in W^{1, p}(\Omega)$ such that $\xi \geq 0$. This implies that $u$ is an "entropy solution" of $\mathrm{S}(\mathrm{f})$ in the sense of [6]. At last, arguing exactly as in [6] (Theorems 8 and 9 ), by doubling the variables, one proves that if $u_{i}$ is a weak solution of $\mathrm{S}\left(\mathrm{f}_{\mathrm{i}}\right)$ for $i=1,2$, with $f_{i} \in L^{1}(\Omega)$, then

$$
\begin{aligned}
\int_{\Omega}\left(u_{1}-u_{2}\right)^{+} \xi & \leq \int_{\Omega}\left(f_{1}-f_{2}\right) \operatorname{Sign}_{0}^{+}\left(u_{1}-u_{2}\right) \xi \\
& \leq \int_{\Omega}\left(f_{1}-f_{2}\right)^{+} \xi,
\end{aligned}
$$

for any $\xi \in W^{1, p}(\Omega)$, and the result follows. We omit here the details of the proof to avoid an unnecessary duplication of arguments.

Remark 1 Notice that if there is no plane region at the 0 level, in other words if $\varphi^{-1}(0)=\{0\}$, then Lemma 2 is not necessary and Lemma 1 with $z$ constant is enough to prove the entropy inequalities for any $k$ and $\xi$ (see [6, Lemma 2 and Theorem 3], for the case $p=2$ ).

\subsection{Proof of the existence}

The proof of the existence employs an approximation argument and standard monotonicity techniques.

Proposition 2 Given $f \in L^{\infty}(\Omega)$ there exists at least one weak solution for the problem (2.3).

Proof. Consider a sequence of increasing continuous functions $\varphi_{n}: \mathbb{R} \rightarrow \mathbb{R}$ such that

$$
\varphi_{n} \longrightarrow \varphi \text { uniformly in the compacts of } \mathbb{R} \text {. }
$$

Using Proposition 2.4 of [3], there exists a unique $u_{n}$ such that

$$
\left\{\begin{array}{l}
u_{n} \in L^{\infty}(\Omega), w_{n}:=\varphi_{n}\left(u_{n}\right) \in W_{0}^{1, p}(\Omega) \text { and } \\
u_{n}=\nabla \cdot \mathbf{a}\left(\nabla \varphi_{n}\left(u_{n}\right), \varphi_{n}\left(u_{n}\right)\right)+f \text { in } \mathcal{D}^{\prime}(\Omega)
\end{array},\right.
$$


and

$$
\left\|u_{n}\right\|_{L^{\infty}(\Omega)} \leq\|f\|_{L^{\infty}(\Omega)},
$$

which, in turn, implies that

$$
\left\|w_{n}\right\|_{L^{\infty}(\Omega)} \leq C \text { (independently of } n \text { ). }
$$

Taking $w_{n}$ as a test function in the equation of (2.11), one gets

$$
\int_{\Omega}\left|\nabla w_{n}\right|^{p}=\int_{\Omega}\left(f-u_{n}\right) w_{n}-\int_{\Omega} F\left(w_{n}\right) \cdot \nabla w_{n}=I_{1}-I_{2} .
$$

It is obvious that

$$
I_{2}=\int_{\Omega} \nabla \cdot\left(\int_{0}^{w_{n}} F(r) \mathrm{d} r\right)=0
$$

and, using (2.13),

$$
I_{1} \leq C \int_{\Omega}|f|
$$

Then, by Poincaré's inequality, we get

$$
\left\|w_{n}\right\|_{W^{1, p}(\Omega)} \leq C\|f\|_{L^{1}(\Omega)},
$$

where $C$ is a constant independent of $n$. Finally, using standard compactness and monotonicity arguments, we find that $u$ satisfies (2.3) and establish the existence result.

Remark 2 For $f \in L^{p^{\prime}}(\Omega)$ it is possible to obtain existence of a weak solution for the problem

$$
\left\{\begin{array}{l}
u \in L^{1}(\Omega), \varphi(u) \in W_{0}^{1, p}(\Omega) \text { and } \\
u-\nabla \cdot \mathbf{a}(\nabla \varphi(u), \varphi(u))=f \text { in } \mathcal{D}^{\prime}(\Omega) .
\end{array}\right.
$$

In fact, let $f_{n}$ be a sequence in $L^{\infty}(\Omega)$, such that

$$
f_{n} \longrightarrow f \text { in } \mathrm{E}^{p^{\prime}}(\Omega), \text { as } n \rightarrow \infty .
$$

It follows from Proposition 2, that there exists a unique $u_{n}$ solution of

$$
\left\{\begin{array}{l}
u_{n} \in L^{\infty}(\Omega), w_{n}:=\varphi\left(u_{n}\right) \in W_{0}^{1, p}(\Omega) \text { and } \\
u_{n}=\nabla \cdot \mathbf{a}\left(\nabla w_{n}, w_{n}\right)+f_{n} \text { in } \mathcal{D}^{\prime}(\Omega)
\end{array}\right.
$$

It is also true that

$$
\left\|w_{n}\right\|_{W^{1, p}(\Omega)} \leq C\left\|f_{n}\right\|_{L^{p^{\prime}}(\Omega)},
$$

for a constant $C$ independent of $n$. Indeed, defining

$$
\varphi_{k}(r)=((\varphi(r) \wedge \varphi(k)) \vee \varphi(-k)
$$


we see that $w_{k, n}:=\varphi_{k}\left(u_{n}\right) \in W_{0}^{1, p}(\Omega) \cap L^{\infty}(\Omega)$ and, as a consequence of $(2.16)$, satisfies

$$
\int_{\Omega}\left|\nabla w_{k, n}\right|^{p}=\int_{\Omega}\left(f_{n}-u_{n}\right) w_{k, n}-\int_{\Omega} F\left(w_{k, n}\right) \cdot \nabla w_{k, n}
$$

It is clear that $\int_{\Omega} u_{n} w_{k, n} \geq 0$ and $\int_{\Omega} F\left(w_{k, n}\right) \cdot \nabla w_{k, n}=0$, so

$$
\begin{aligned}
\int_{\Omega}\left|\nabla w_{k, n}\right|^{p} & \leq \int_{\Omega} f_{n} w_{k, n} \\
& \leq\left\|f_{n}\right\|_{L^{p^{\prime}}(\Omega)}\left\|w_{k, n}\right\|_{L^{p}(\Omega)}
\end{aligned}
$$

and taking into account Poincaré's inequality we get

$$
\left\|\nabla w_{k, n}\right\|_{L^{p}(\Omega)} \leq C\left\|f_{n}\right\|_{L^{p^{\prime}(\Omega)}} .
$$

Then letting $k \rightarrow \infty$ and using Poincaré's inequality again, we obtain (2.17). Taking $f_{n}, f_{m} \in L^{\infty}(\Omega)$ and using Proposition 1, we get

$$
\left\|u_{n}-u_{m}\right\|_{L^{1}(\Omega)} \leq\left\|f_{n}-f_{m}\right\|_{L^{1}(\Omega)}
$$

so, $\left(u_{n}\right)$ is a Cauchy sequence in $L^{1}(\Omega)$ and there exists $u \in L^{1}(\Omega)$ such that $u_{n} \rightarrow u$ in $L^{1}(\Omega)$, as $n \rightarrow \infty$. Thanks to $(2.17)$, we have

$$
w_{n} \longrightarrow w=\varphi(u) \text { weakly in } W^{1, p} \text { and strongly in } L^{p}(\Omega)
$$

and letting $n \rightarrow \infty$ in the equation satisfied by $u_{n}$, we conclude that $u$ is a solution of (2.15). The uniqueness for this problem is left open.

The proof of Theorem 1 is now a direct consequence of Propositions 1 and 2 .

\section{The evolution problem}

We now turn our attention to the evolution problem

$$
\left\{\begin{array}{lll}
u_{t}=\Delta_{p} \varphi(u)+\nabla \cdot F(\varphi(u))+h & \text { in } Q:=(0, T) \times \Omega & \\
\varphi(u)=0 & \text { on } \Sigma:=(0, T) \times \Gamma, & \mathrm{E}\left(\mathrm{u}_{0}, \mathrm{~h}\right) \\
u(0, .)=u_{0}(.) & \text { in } \Omega
\end{array}\right.
$$

where $\varphi$ and $F$ satisfy (2.1) and (2.2), respectively. The notion of weak solution we have in mind is the following:

Definition 1 Given $u_{0} \in L^{\infty}(\Omega)$ and $h \in L^{\infty}(Q)$, a weak solution of $\mathrm{E}\left(\mathrm{u}_{0}, \mathrm{~h}\right)$ is a function $u$ such that

$$
\left\{\begin{array}{l}
u \in L^{\infty}(Q), \varphi(u) \in L^{p}\left(0, T ; W_{0}^{1, p}(\Omega)\right) \\
-\iint_{Q} u \xi_{t}+\iint_{Q}\left(|\nabla \varphi(u)|^{p-2} \nabla \varphi(u)+F(\varphi(u))\right) \cdot \nabla \xi \\
=\iint_{Q} h \xi+\int_{\Omega} u_{0} \xi(0), \forall \xi \in V_{0}^{p}
\end{array}\right.
$$


where

$$
V_{0}^{p}=\left\{\xi \in L^{p}\left(0, T ; W^{1, p}(\Omega)\right) ; \xi_{t} \in L^{1}(Q), \xi(T) \equiv 0\right\} .
$$

We shall prove the following result:

Theorem 2 For any $u_{0} \in L^{\infty}(\Omega)$ and $h \in L^{\infty}(Q)$, there exists a unique weak solution $u$ of $\mathrm{E}\left(\mathrm{u}_{0}, \mathrm{~h}\right)$. Moreover, $u \in C\left([0, T) ; L^{1}(\Omega)\right), u(0)=u_{0}$ and if $u_{0 i} \in$ $L^{\infty}(\Omega)$ and $h_{i} \in L^{\infty}(Q)$ and $u_{i}$ are the corresponding weak solutions of $\mathrm{E}\left(\mathrm{u}_{0 \mathrm{i}}, \mathrm{h}_{\mathrm{i}}\right)$, for $i=1,2$, then

$$
\int_{\Omega}\left(u_{1}(t)-u_{2}(t)\right)^{+} \leq \int_{\Omega}\left(u_{01}-u_{02}\right)^{+}+\int_{0}^{t} \int_{\Omega}\left(h_{1}-h_{2}\right)^{+} .
$$

\subsection{Proof of the uniqueness}

The proof of the uniqueness for the evolutionary problem follows the same general ideas used in the stationary case. It is obtained for data in $L^{1}$ so that the space of test functions we have in mind in this section is the subspace of $V_{0}^{p}$ for which all the integrals in the definition have a meaning in this context. The result is the following:

Proposition 3 Let $h_{1}, h_{2} \in L^{1}(\Omega)$ and $u_{01}, u_{02} \in L^{1}(\Omega)$. If $u_{i}$ is a weak solution of $\mathrm{E}\left(\mathrm{u}_{0 \mathrm{i}}, \mathrm{h}_{\mathrm{i}}\right)$ for $i=1,2$, then, for a.e. $t \in(0, T)$,

$$
\int_{\Omega}\left(u_{1}(t)-u_{2}(t)\right)^{+} \leq \int_{\Omega}\left(u_{01}-u_{02}\right)^{+}+\int_{0}^{t} \int_{\Omega}\left(h_{1}-h_{2}\right)^{+} .
$$

We postpone the proof of the proposition and start by obtaining entropy inequalities (for functions $z$ ) outside the set where $\varphi$ is constant.

Lemma 4 Consider $u_{0} \in L^{1}(\Omega)$ and $h \in L^{1}(Q)$ and let $u$ be a weak solution of $\mathrm{E}\left(\mathrm{u}_{0}, \mathrm{~h}\right)$. Then

$$
\begin{aligned}
& \iint_{Q} \operatorname{Sign}_{0}^{+}(u-z)\left\{(u-z) \xi_{t}-(\mathbf{a}(\nabla \varphi(u), \varphi(u))-\mathbf{a}(\nabla \varphi(z), \varphi(z))) \cdot \nabla \xi\right\} \\
& \geq \iint_{Q} \operatorname{Sign}_{0}^{+}(u-z) \xi \nabla \cdot \mathbf{a}(\nabla \varphi(z), \varphi(z))-\int_{\Omega}\left(u_{0}-z\right)^{+} \xi(0) \\
& \quad-\iint_{Q} \operatorname{Sign}_{0}^{+}(u-z) h \xi
\end{aligned}
$$

for all $(z, \xi) \in L^{\infty}(\Omega) \times\left[W^{1, p}(Q) \cap L^{\infty}(Q) \cap C\left([0, T] ; L^{\infty}(\Omega)\right)\right]$ such that $\xi \geq 0$, $\xi(T,)=$.0 a.e. in $\Omega, \varphi(z) \in W^{1, p}(\Omega), \varphi(z) \notin E$ a.e. in $\Omega, \nabla \cdot \mathbf{a}(\nabla \varphi(z), \varphi(z)) \in$ $L^{1}(\Omega)$ and $(\varphi(u)-\varphi(z))^{+} \xi=0$ on $\Sigma$. 
Proof. Let $(z, \xi)$ be as in the statement of the lemma. Using an idea of [3] (cf. Theorem 4.4), we extend $u$ onto $\mathbb{R} \times \Omega$ by 0 if $t>T$ and by $u_{0}$ if $t<0$. Note that then

$$
\Phi=H_{\varepsilon}(\varphi(u)-\varphi(z)) \xi \in L^{p}\left(0, T ; W_{0}^{1, p}(\Omega)\right)
$$

so, for any $h>0, \Phi^{h}(t)=\frac{1}{h} \int_{t}^{t+h} \Phi(s) d s$ is an admissible test function in the problem and

$$
\iint_{Q}\left(u-u_{0}\right) \Phi_{t}^{h}-\iint_{Q} \mathbf{a}(\nabla \varphi(u), \varphi(u)) \cdot \nabla \Phi^{h}+\iint_{Q} h \Phi^{h}=0 .
$$

We see that

$$
\begin{aligned}
\iint_{Q}\left(u-u_{0}\right) \Phi_{t}^{h} & =\iint_{Q}\left(u-u_{0}\right) \frac{\Phi(t+h)-\Phi(t)}{h} \\
& =\iint_{Q} \frac{u(t-h)-u(t)}{h} \Phi(t)
\end{aligned}
$$

and, since for any $r, \hat{r}, w \in \mathbb{R}$,

$$
(\hat{r}-r) H_{\varepsilon}(\varphi(r)-\varphi(w)) \leq \psi_{w}^{\epsilon}(\hat{r})-\psi_{w}^{\epsilon}(r)
$$

where

it follows that

$$
\psi_{w}^{\epsilon}(r)=\int_{w}^{r} H_{\varepsilon}(\varphi(s)-\varphi(w)) \mathrm{d} s
$$

$$
\begin{aligned}
& \iint_{Q}\left(u-u_{0}\right) \Phi_{t}^{h} \leq \iint_{Q} \frac{\psi_{z(t, x)}^{\epsilon}(u(t-h, x))-\psi_{z(t, x)}^{\epsilon}(u(t, x))}{h} \xi(t, x) d t d x \\
& \quad \leq \iint_{Q}\left(\psi_{z(t, x)}^{\epsilon}(u(t, x))-\psi_{z(t, x)}^{\epsilon}\left(u_{0}(x)\right)\right) \frac{\xi(t+h, x)-\xi(t, x)}{h} d t d x .
\end{aligned}
$$

Consequently, we have

$$
\begin{aligned}
\liminf _{h \rightarrow 0} \iint_{Q}\left(u-u_{0}\right) \Phi_{t}^{h} & \leq \iint_{Q}\left(\psi_{z}^{\epsilon}(u)-\psi_{z}^{\epsilon}\left(u_{0}\right)\right) \xi_{t} \\
& \leq \iint_{Q} \xi_{t}\left\{\int_{u_{0}}^{u} H_{\varepsilon}(\varphi(s)-\varphi(z)) \mathrm{d} s\right\} .
\end{aligned}
$$

Since $\Phi^{h} \rightarrow H_{\varepsilon}(\varphi(u)-\varphi(z)) \xi$ in $L^{p}\left(0, T ; W_{0}^{1, p}(\Omega)\right)$, from (3.4) and the preceding estimate it follows that

$$
\begin{aligned}
& \iint_{Q} \xi_{t}\left\{\int_{u_{0}}^{u} H_{\varepsilon}(\varphi(s)-\varphi(z)) \mathrm{d} s\right\}-\iint_{Q} \mathbf{a}(\nabla \varphi(u), \varphi(u)) \cdot \nabla\left(H_{\varepsilon}(\varphi(u)-\varphi(z)) \xi\right) \\
& \geq-\iint_{Q} H_{\varepsilon}(\varphi(u)-\varphi(z)) h \xi .
\end{aligned}
$$


It is clear that, as $\varepsilon \rightarrow 0$,

$$
\begin{aligned}
\int_{u_{0}(x)}^{u(t, x)} H_{\varepsilon}(\varphi(s)-\varphi(z(x))) d s & \longrightarrow \int_{u_{0}(x)}^{u(t, x)} \operatorname{Sign}_{0}^{+}(\varphi(s)-\varphi(z(x))) d s \\
& =\int_{u_{0}(x)}^{u(t, x)} \operatorname{Sign}_{0}^{+}(s-z(x)) \mathrm{d} s \\
& =(u(t, x)-z(x))^{+}-\left(u_{0}(x)-z(x)\right)^{+}
\end{aligned}
$$

a.e. $(t, x) \in Q$. Moreover,

$$
\left|\int_{u_{0}(x)}^{u(t, x)} H_{\varepsilon}(\varphi(s)-\varphi(z(x))) \mathrm{d} s\right| \leq\left|u(t, x)-u_{0}(x)\right|,
$$

so, by Lebesgue's dominated convergence theorem, the first term in (3.5) converges to

$$
\iint_{Q}(u(t, x)-z(x))^{+} \xi_{t}+\int_{\Omega}\left(u_{0}(x)-z(x)\right)^{+} \xi(0) .
$$

It is obvious that the last term converges to

$$
\iint_{Q} \operatorname{Sign}_{0}^{+}(u-z) h \xi
$$

and concerning the second one we proceed exactly as in Lemma 1, concluding the proof.

We now derive the entropy inequalities for constants belonging to the set where $\varphi$ is constant and equal to 0 .

Lemma 5 Assume that $0 \in E$ and that $\varphi^{-1}(0)=[m, M]$ is bounded. Take $h \in$ $L^{1}(Q)$ and $u_{0} \in L^{1}(\Omega)$ and let $u$ be a weak solution of $E\left(u_{0}, h\right)$. Then

$$
\begin{gathered}
\iint_{Q} \operatorname{Sign}_{0}^{+}(u-k)\left\{(u-k) \xi_{t}-\mathbf{a}(\nabla \varphi(u), \varphi(u)) \cdot \nabla \xi\right\} \\
\geq-\int_{\Omega}\left(u_{0}-k\right)^{+} \xi(0)-\iint_{Q} \operatorname{Sign}_{0}^{+}(u-k) h \xi
\end{gathered}
$$

for any $(k, \xi) \in \mathbb{R} \times\left[W^{1, p}(Q) \cap L^{\infty}(Q) \cap C\left([0, T] ; L^{\infty}(\Omega)\right)\right]$ such that $\xi \geq 0$, $\xi(T,)=$.0 a.e. in $\Omega$ and $m \leq k \leq M$.

Proof. To start with observe that taking a sequence $\left(k_{n}\right)_{n \in \mathbb{N}}$ such that $M<k_{n}$, $\varphi\left(k_{n}\right) \notin E$ and $k_{n} \downarrow M$ we get

$$
\operatorname{Sign}_{0}^{+}\left(u-k_{n}\right) \uparrow \operatorname{Sign}_{0}^{+}(u-M) \quad \text { and } \operatorname{Sign}_{0}^{+}\left(u_{0}-k_{n}\right) \uparrow \operatorname{Sign}_{0}^{+}\left(u_{0}-M\right)
$$


a.e. in $\Omega$; hence, replacing $z$ by $k_{n}$ in (3.3) and letting $n \rightarrow \infty$, we arrive at

$$
\begin{gathered}
\iint_{Q} \operatorname{Sign}_{0}^{+}(u-M)\left\{(u-M) \xi_{t}-\mathbf{a}(\nabla \varphi(u), \varphi(u)) \cdot \nabla \xi\right\} \\
\geq-\int_{\Omega}\left(u_{0}-M\right)^{+} \xi(0)-\iint_{Q} \operatorname{Sign}_{0}^{+}(u-M) h \xi .
\end{gathered}
$$

On the other hand, let $z_{n}$ be given as in Lemma 2 and use Lemma 4 to conclude that

$$
\begin{aligned}
& \iint_{Q}\left(u-z_{n}\right)^{+} \xi_{t}-\operatorname{Sign}_{0}^{+}\left(u-z_{n}\right)\left\{\left(\mathbf{a}(\nabla \varphi(u), \varphi(u))-\mathbf{a}\left(\nabla \varphi\left(z_{n}\right), \varphi\left(z_{n}\right)\right)\right) \cdot \nabla \xi\right\} \\
& \geq-\int_{\Omega}\left(u_{0}-z_{n}\right)^{+} \xi(0)+\iint_{Q} \operatorname{Sign}_{0}^{+}\left(u-z_{n}\right) \xi \nabla \cdot \mathbf{a}\left(\nabla \varphi\left(z_{n}\right), \varphi\left(z_{n}\right)\right) . \\
& \quad-\iint_{Q} \operatorname{Sign}_{0}^{+}\left(u-z_{n}\right) h \xi .
\end{aligned}
$$

Since it is clear that $\operatorname{Sign}_{0}^{+}\left(u-z_{n}\right) \downarrow \operatorname{Sign}_{\text {max }}^{+}(u-m)$ as $n \rightarrow \infty$ and that

$$
\operatorname{Sign}_{m a x}^{+}(u-m) \mathbf{a}(\nabla \varphi(u), \varphi(u))=\operatorname{Sign}_{0}^{+}(u-M) \mathbf{a}(\nabla \varphi(u), \varphi(u)),
$$

by letting $n \rightarrow \infty$ in (3.7), one obtains

$$
\begin{array}{r}
\iint_{Q}(u-m)^{+} \xi_{t}-\operatorname{Sign}_{0}^{+}(u-M) \mathbf{a}(\nabla \varphi(u), \varphi(u)) \cdot \nabla \xi \\
\geq-\int_{\Omega}\left(u_{0}-m\right)^{+} \xi(0)-\iint_{Q} \operatorname{Sign}_{\max }^{+}(u-m) h \xi
\end{array}
$$

Now, we extend $\xi$ (resp. $u$ ) onto $(-1 / 2, T) \times \Omega$ as a $W^{1, p}$ function such that $\xi(-1 / 2) \equiv 0$ (resp. $u=u_{0}$ for $-1 / 2 \leq t \leq 0$ ), and apply the modified version of Lemma 2 of [6], with $\mathcal{O}=(-1 / 2, T) \times \Omega$,

$$
\left\{\begin{array}{l}
F=\operatorname{Sign}_{0}^{+}(u-M) \mathbf{a}(\nabla \varphi(u), \varphi(u)), \quad G_{1}=\operatorname{Sign}_{0}^{+}(u-M) h \\
G_{2}=\operatorname{Sign}_{\text {max }}^{+}(u-m) h \text { and } e=(-1,0)
\end{array}\right.
$$

a.e. in $(0, T) \times \Omega$ and

$$
F=G_{1}=G_{2}=0 \quad \text { in } \quad(-1 / 2,0] \times \Omega .
$$

Consequently

$$
\begin{aligned}
& \iint_{Q}\left((u-k)^{+}-\left(u_{0}-k\right)^{+}\right) \xi_{t}-\operatorname{Sign}_{0}^{+}(u-M) \mathbf{a}(\nabla \varphi(u), \varphi(u)) \cdot \nabla \xi \\
& \quad \geq \int_{\Omega}\left(\operatorname{Sign}_{0}^{+}(u-M)(1-\tilde{H}(u-k))+\operatorname{Sign}_{m a x}^{+}(u-m) \tilde{H}(u-k)\right) h \xi
\end{aligned}
$$


for all $k \in[m, M]$, for some $\tilde{H}(u-k) \in \operatorname{Sign}^{+}(u-k)$. Since

$$
\operatorname{Sign}_{0}^{+}(u-M)(1-\tilde{H}(u-k))=0
$$

and

$$
\operatorname{Sign}_{\text {max }}^{+}(u-m) \tilde{H}(u-k)=\tilde{H}(u-k)
$$

we get

$$
\begin{aligned}
& \iint_{Q}(u-k)^{+} \xi_{t}-\operatorname{Sign}_{0}^{+}(u-k) \mathbf{a}(\nabla \varphi(u), \varphi(u)) \cdot \nabla \xi \\
& \geq-\int_{\Omega}\left(u_{0}-k\right)^{+} \xi(0)-\int_{\Omega} h \xi \tilde{H}(u-k)
\end{aligned}
$$

and conclude exactly as in the proof of Lemma 3, obtaining (3.6).

The two lemmas allow us to prove Proposition 3 using the same reasoning applied in the proof of Proposition 1. We have to use Theorems 13 and 14 of [6].

Remark 3 We remark that the preceding arguments apply to more general equations of the form

$$
u_{t}=\operatorname{div} \mathbf{a}(x, \nabla \varphi(u), \varphi(u))+h \quad \text { in } Q, \quad \varphi(u)=0 \text { on } \Sigma,
$$

with a satisfying appropriate structure assumptions, to prove that weak solutions satisfy entropy inequalities of the type of (3.3) and (3.6). However, the process of doubling the variables, that is essential to show that entropy solutions are unique, becomes very complicated in such a generality. For some particular cases, like

$$
\mathbf{a}(x, \eta, r)=b(\eta)+G(x) r,
$$

with $b$ a monotone function in $\mathbb{R}^{N}$ and $G \in\left[W^{1, \infty}(\Omega)\right]^{N}$, the arguments of [6] and [8] (see the proof on page 152) may be adapted to prove the uniqueness of entropy solutions, so the results of Propositions 1 and 3 remain true under the condition (3.9).

Remark 4 We conclude this section by showing that, in general, uniqueness for the weak solution of (1.1) is not to be expected. Consider the one-dimensional problem in $(t, x) \in Q=(0,1) \times \Omega, \Omega=(0,1)$

$$
\left\{\begin{array}{l}
u_{t}=\varphi(u)_{x x}+u_{x} \\
\varphi(u)_{\mid \partial \Omega}=0 \\
u(0)=1
\end{array}\right.
$$

with $\varphi^{-1}(s)=s+H(s)$, where $H$ is the Heaviside graph. A weak solution is a function $u \in L^{\infty}(Q)$ such that $\varphi(u) \in L^{2}\left(0,1 ; H_{0}^{1}(\Omega)\right)$ and $u$ solves the equation in the sense of distributions, i.e., for a suitable space of test functions $\mathcal{T}$,

$$
-\int_{0}^{1} \int_{0}^{1} u \xi_{t}+\int_{0}^{1} \int_{0}^{1} \varphi(u)_{x} \xi_{x}+\int_{0}^{1} \int_{0}^{1} u \xi_{x}=\int_{0}^{1} \xi(0), \forall \xi \in \mathcal{T} .
$$


It is obvious that the constant function $u_{1} \equiv 1$ is a weak solution. Let's construct a different one; take the $C^{1}$ function $F$ defined in $[0,2]$ by

$$
F(s)= \begin{cases}1 & \text { if } 0 \leq s \leq 1 \\ 1-(s-1)^{2} & \text { if } 1<s \leq 2\end{cases}
$$

and put $u_{2}(t, x)=F(t+x)$. We have $\left(u_{2}\right)_{t}(t, x)=F^{\prime}(t+x)\left(u_{2}\right)_{x}(t, x)$ and $u_{2}(0, x)=F(x)=1$ because $x \in(0,1)$. Since $0 \leq F \leq 1$, we conclude that $u_{2}$ is also a weak solution and it is of course different from $u_{1}$. So there is no uniqueness for the problem.

\subsection{Proof of the existence}

Let's define in $L^{1}(\Omega)$ an operator $A$ by

$$
A z=-\Delta_{p} \varphi(z)-\nabla \cdot F(\varphi(z)) \text { in } \mathcal{D}^{\prime}(\Omega)
$$

with domain

$$
\mathcal{D}(A)=\left\{z \in L^{\infty}(\Omega): \varphi(z) \in W_{0}^{1, p}(\Omega) \text { and } A z \in L^{1}(\Omega)\right\} .
$$

It follows from Theorem 1 that $A$ is T-accretive and from Proposition 2 that

$$
R(I+\lambda A) \supseteq L^{\infty}(\Omega), \text { for all } \lambda>0 .
$$

Moreover, using the same proof as in [3] (cf. Proposition 2.4. iii), step 3) we have the following result:

Lemma 6 The domain $\mathcal{D}(A)$ of the operator $A$ is dense in $L^{1}(\Omega)$, i.e.,

$$
\overline{\mathcal{D}(A)}^{L^{1}(\Omega)}=L^{1}(\Omega) .
$$

We can now use the general theory of evolution equations (see e.g. [2] and [7]), to obtain, for any $u_{0} \in L^{1}(\Omega)$ and $h \in L^{1}(Q)$, a unique mild solution $u \in \mathcal{C}\left([0, T) ; L^{1}(\Omega)\right)$ of

$$
\frac{d u}{d t}+A u=h \quad \text { on } \quad(0, T), \quad u(0)=u_{0} .
$$

We show that this mild solution is also a weak solution of $E\left(u_{0}, h\right)$, thus obtaining

Proposition 4 Given $u_{0} \in L^{\infty}(\Omega)$ and $h \in L^{\infty}(Q)$ there exists at least one weak solution $u$ of $E\left(u_{0}, h\right)$.

Proof. By definition of mild solution, $u(t)=L^{1}-\lim u_{\varepsilon}(t)$ uniformly in $t \in[0, T]$, where for $\varepsilon>0, u_{\varepsilon}$ is a $\varepsilon$-approximate solution corresponding to a subdivision $t_{0}=0<t_{1}<\ldots<t_{n-1}<T \leq t_{n}$, with $t_{i}-t_{i-1}=\varepsilon$ and $h_{1}, \ldots h_{n} \in L^{\infty}(\Omega)$ with 
$\sum_{i=1}^{n} \int_{t_{i-1}}^{t_{i}}\left\|h(t)-h_{i}\right\|_{L^{1}} d t \leq \varepsilon$. This approximate solution is defined by $u_{\varepsilon}(0)=u_{0}$ and $u_{\varepsilon}(t)=u_{i}$ for $\left.\left.t \in\right] t_{i-1}, t_{i}\right]$, where $u_{i} \in L^{\infty}(\Omega)$ satisfies

$$
\frac{u_{i}-u_{i-1}}{\epsilon}+A u_{i}=h_{i}
$$

that is $w_{i}:=\varphi\left(u_{i}\right) \in W_{0}^{1, p}(\Omega)$ and by density, for all $\xi \in W_{0}^{1, p}(\Omega)$, we have

$$
\int_{\Omega}\left(\left|\nabla w_{i}\right|^{p-2} \nabla w_{i}+F\left(w_{i}\right)\right) \cdot \nabla \xi=\int_{\Omega} h_{i} \xi-\int_{\Omega} \frac{u_{i}-u_{i-1}}{\epsilon} \xi .
$$

It follows that

$$
\left\|u_{i}\right\|_{L^{\infty}(\Omega)} \leq\left\|u_{0}\right\|_{L^{\infty}(\Omega)}+i \varepsilon\left\|h_{i}\right\|_{L^{\infty}(\Omega)},
$$

so that

$$
\left\|u_{\varepsilon}(t)\right\|_{L^{\infty}(\Omega)} \leq M_{1}:=\left\|u_{0}\right\|_{L^{\infty}(\Omega)}+T\|h\|_{L^{\infty}(\Omega)}, \quad \forall t \in[0, T] .
$$

Taking $\xi=w_{i}$ in $(3.10)$, one gets

$$
\varepsilon \int_{\Omega}\left|\nabla w_{i}\right|^{p} \leq \varepsilon \int_{\Omega} h_{i} w_{i}-\int_{\Omega}\left(u_{i}-u_{i-1}\right) w_{i}-\varepsilon \int_{\Omega} F\left(w_{i}\right) \cdot \nabla w_{i} .
$$

We can see as before that the last integral vanishes and also that

$$
\left(u_{i-1}-u_{i}\right) w_{i} \leq \psi\left(u_{i-1}\right)-\psi\left(u_{i}\right)
$$

where $\psi(r)=\int_{0}^{r} \varphi(s) \mathrm{d} s$, so (3.12) implies

$$
\varepsilon \int_{\Omega}\left|\nabla w_{i}\right|^{p}+\int_{\Omega} \psi\left(u_{i}\right) \leq \varepsilon \varphi\left(M_{1}\right) \int_{\Omega}\left|h_{i}\right|+\int_{\Omega} \psi\left(u_{i-1}\right) .
$$

Adding (3.13), for $i=1$ to $n$, we get

$$
\varepsilon \sum_{i=1}^{n} \int_{\Omega}\left|\nabla w_{i}\right|^{p}+\int_{\Omega} \psi\left(u_{n}\right) \leq \varepsilon \varphi\left(M_{1}\right) \sum_{i=1}^{n} \int_{\Omega}\left|h_{i}\right|+\int_{\Omega} \psi\left(u_{0}\right),
$$

so that $w_{\varepsilon}:=\varphi\left(u_{\varepsilon}\right)$ satisfies

$$
\begin{aligned}
\int_{0}^{T} \int_{\Omega}\left|\nabla w_{\varepsilon}\right|^{p} & =\varepsilon \sum_{i=1}^{n} \int_{\Omega}\left|\nabla w_{i}\right|^{p} \\
& \leq \varphi\left(M_{1}\right)\left\{\|h\|_{L^{1}(Q)}+\varepsilon\right\}+\int_{\Omega} \psi\left(u_{0}\right)
\end{aligned}
$$

It follows from (3.11) and Poincaré's inequality that $w_{\varepsilon}$ is a bounded sequence in $L^{p}\left(0, T ; W_{0}^{1, p}(\Omega)\right)$ and that $\left|\nabla w_{\epsilon}\right|^{p-2} \nabla w_{\epsilon}$ is bounded in $L^{p^{\prime}}(\Omega)$. Choose a subsequence such that $w_{\varepsilon_{k}} \rightarrow w$ in $L^{p}\left(0, T ; W_{0}^{1, p}(\Omega)\right)$ and

$$
\left|\nabla w_{\epsilon}\right|^{p-2} \nabla w_{\epsilon} \rightarrow G
$$


weakly in $L^{p^{\prime}}(\Omega)$; since $u_{\varepsilon} \rightarrow u$ in $L^{1}(Q)$ and $w_{\varepsilon}=\varphi\left(u_{\varepsilon}\right)$, a.e. in $Q$, in the limit we obtain $w=\varphi(u)$, a.e. in $Q$. At last, let $\tilde{u}_{\varepsilon}$ be the function defined from $[0, T]$ into $L^{1}(\Omega)$ by $\tilde{u}_{\varepsilon}\left(t_{i}\right)=u_{i}$ and $\tilde{u}_{\varepsilon}$ is linear in $\left[t_{i-1}, t_{i}\right]$. Then

$$
\tilde{u}_{\epsilon t}=\Delta_{p} w_{\varepsilon}+\nabla \cdot F\left(w_{\varepsilon}\right)+h_{\varepsilon} \text { in } \mathcal{D}^{\prime}(Q),
$$

where $h_{\epsilon}$ is the function from $[0, T]$ into $L^{1}(\Omega)$ defined by $h_{\varepsilon}(t)=h_{i}$ for $\left.\left.t \in\right] t_{i-1}, t_{i}\right]$ and $i=1, \ldots, n$. It is clear that $\tilde{u}_{\epsilon} \rightarrow u$ and $h_{\epsilon} \rightarrow h$ in $L^{1}(\Omega)$ as $\epsilon \rightarrow 0$ and, as in the proof of Proposition 2, we can use a monotonicity argument to show that $G=|\nabla w|^{p-2} \nabla w$, a.e. in $Q$. Then letting $\epsilon \rightarrow 0$ one gets that $u$ is a weak solution of $E\left(u_{0}, h\right)$.

Theorem 2 is a direct consequence of Propositions 3 and 4 .

Acknowledgements The authors would like to thank Philippe Bénilan and José Carrillo for stimulating discussions on this subject. This paper was written while the first author was a postdoctoral student at the C.M.A.F./Universidade de Lisboa and part of the results were obtained while he visited the Departamento de Matemática da Universidade de Coimbra. He is grateful to both institutions for their hospitality.

\section{References}

[1] H.W. ALT, S. LUCKHAUS, Quasilinear elliptic-parabolic differential equations, Math. Z. 183 (1983), 311-341.

[2] PH. BÉNILAN, Equations d'évolution dans un espace de Banach quelconque et applications, Thèse d'état, Orsay 1972.

[3] PH. BÉNILAN, P. WITTBOLD, On mild and weak solutions of ellipticparabolic problems, Adv. Differential Equations 1 (1996), 1053-1073.

[4] PH. BÉNILAN, P. WITTBOLD, Sur un problème parabolique-elliptique, M2AN Math. Model. Numer. Anal. 33 (1999), 121-127.

[5] H. BRÉZIS, M. CRANDALL, Uniqueness of solutions of the initial-value problem for $u_{t}-\Delta \varphi(u)=0$, J. Math. Pures Appl. 58 (1979), 153-163.

[6] J. CARRILLO, Entropy solutions for nonlinear degenerate problems, Arch. Rat. Mech. Anal. 147 (1999), 269-361.

[7] M. CRANDALL, T. LIGGETT, Generation of semi-groups of nonlinear transformations in general Banach spaces, Amer. J. Math. 93 (1971), $265-298$. 
[8] G. GAGNEUX, M. MADAUNE-TORT, Analyse mathématique de modèles non linéaires de l'ingénierie pétrolière, Mathématiques et Applications, Springer-Verlag, Berlin 22 (1996).

[9] J. KAČUR, On a solution of degenerate elliptic-parabolic systems in OrliczSobolev spaces I-II, Math. Z. 203 (1990), 153-171 and 569-579.

[10] J. KAČUR, Solution of some free boundary problems by relaxation schemes, SIAM J. Numer. Anal. 36 (1999), 290-316.

[11] S. KAMENOMOSTKAYA, On the Stefan problem (in Russian), Naučnye Dokl. Vyš̌ei Školy 1 (1958), 60-62; Mat. Sb. 53 (95) (1961), 489-514.

[12] F. OTTO, $L^{1}$-contraction and uniqueness for quasilinear elliptic-parabolic equations, J. Differential Equations 131 (1996), 20-38.

[13] J.F. RODRIGUES, Variational methods in the Stefan problem, in: Phase Transitions and Hysteresis (A. Visintin, Ed.), 147-212, Lect. Notes Math. Springer-Verlag, Berlin 1584 (1994).

[14] J.M. URBANO, A free boundary problem with convection for the p-Laplacian, Rend. Mat. Appl. 17 (1997), 1-19.

[15] J.M. URBANO, Continuous solutions for a degenerate free boundary problem, Ann. Mat. Pura Appl. 178 (2000), 195-224.

[16] J.L. VAZQUEZ, A strong maximum principle for some quasilinear elliptic equations, Appl. Math. Optim. 12 (1984), 191-201.

(D) To access this journal online:

(20) http://www.birkhauser.ch 